\title{
Uso do Método de Monte Carlo para caracterização adicional de materiais para aplicação em blindagem de fótons
} Using Monte Carlo Method for additional characterization of
materials for photons shielding application

\author{
Natalia Amaral Lourenço ${ }^{1}$, Gabriela Hoff², Iana Borba², Maikon Moreira de Pires ${ }^{3}$, \\ Chiara das Dores do Nascimento ${ }^{3}$, Everton Granemann Souza ${ }^{3}$, Tiago Severo Garcia ${ }^{4}$, \\ Henrique Trombini ${ }^{1}$, Luis Gustavo Pereira ${ }^{1}$
}

\begin{abstract}
${ }^{1}$ Departamento de Física - Universidade Federal do Rio Grande do Sul, Porto Alegre, Brasil ${ }^{2}$ Serviço de Física Médica e Radioproteção - Hospital de Clínicas de Porto Alegre, Porto Alegre, Brasil ${ }^{3}$ Programa de Pós-Graduação em Engenharia Eletrônica e Computação - Universidade Católica de Pelotas, Pelotas, Brasil ${ }^{4}$ Serviço de Radiologia - Hospital de Clínicas de Porto Alegre, Porto Alegre, Brasil
\end{abstract}

\begin{abstract}
Resumo
As blindagens são uma parte fundamental na radioproteção. A construção de curvas de transmissão para um material utilizado como blindagem é importante para a definição da espessura necessária da blindagem. O principal objetivo deste trabalho é desenvolver um método para caracterizar novos materiais para blindagem de fótons usando a ferramenta computacional Geant4. As amostras irradiadas, denominadas Traço A, B e C, foram produzidas no Laboratório de Resistência de Materiais da UCPel. As validações experimentais de caracterização das amostras foram realizadas pelo teste da camada semirredutora (CSR), medida de kerma e medidas de Rutherford Backscattering Spectrometry (RBS), sendo os dois primeiros validados por meio de simulações. Os resultados de RBS e do imageamento mostraram a influência da granularidade dos agregados do concreto na homogeneidade e reprodutibilidade das amostras. A validação da aplicação foi feita por meio da verificação dos espectros teóricos e simulados, da geometria de radiação e caracterização do material. Os espectros teóricos e simulados foram validados por testes estatísticos, sendo considerados equivalentes. A geometria de radiação foi validada utilizando um visualizador e as ferramentas de verificação da geometria disponibilizadas pelo Geant4. Espera-se, com esses resultados, fazer medidas experimentais e criar curvas de transmissão simuladas para aplicações na radiologia diagnóstica e radioterapia.
\end{abstract}

Palavras-chave: radiologia diagnóstica; camada semirredutora; Geant4; blindagem.

\begin{abstract}
$X$-rays shieldings are a fundamental part of radioprotection. The built of the transmission curves for a material used as shielding is important in defining the required shielding thickness. The main goal of this work is to develop a method to characterize new materials for photon shielding using the computation tool Geant4. The irradiated samples, called "Traço A", "Traço B" and "Traço C", were produced at the Materials Resistance Laboratory of UCPel. Experimental validations of sample characterization were performed using the half-value layer (HVL), kerma measurements and Rutherford Backscattering Spectrometry (RBS) measures, the first two being validated by simulations. RBS results and imaging tests showed the influence of the granularity of concrete aggregates on the homogeneity and reproducibility of the samples. Application validation was performed by comparing theoretical and simulated spectra, radiation geometry and material characterization. Theoretical and simulated spectra were validated by statistical tests being considered equivalent. Radiation geometry was validated using a viewer and geometry verification tools provided by Geant4. With these results, it is expected to make experimental measures and create simulated transmission curves for radiology and radiotherapy applications.
\end{abstract} Keywords: diagnostic radiology; half-value layer; Geant4; shielding.

\section{Introdução}

As barreiras de proteção (denominadas blindagens) são uma das ferramentas utilizadas para atenuar a exposição excessiva à radiação ionizante, otimizando seu uso para somente o indispensável. Em aplicações médicas, tem-se como objetivo a segurança no cotidiano de profissionais ocupacionalmente expostos, de pacientes e de pessoas que circulam ao redor da instituição hospitalar/clínica. A proteção radiológica é necessária para minimizar possíveis danos causados pela exposição e/ou contaminação da radiação ionizante proveniente de aparelhos eletromédicos emissores de raios $X$ ou materiais radioativos $(1,2)$. Sendo assim, as blindagens são objetos de estudo frequentes (3), devido a necessidade de construir blindagens cada vez mais eficientes, bem como otimizar o material utilizado para reduzir custos na construção civil das mesmas. A otimização da barreira, os métodos ou técnicas de imageamento são ações comuns e refletem, na prática diária, a aplicação do princípio ALARA (4-7) (As Low As Reasonably Achievable). O princípio ALARA pode ser atingido através da aplicação de ações simples, conhecidas como regras básicas da proteção radiológica (8,9): (i) expor-se o menor tempo necessário à radiação ionizante, (ii) manter a maior distância possível da fonte de radiação $X$ e, se preciso, (iii) utilizar barreiras entre a fonte e o ente de proteção. A redução no tempo de exposição e aumento da distância até a fonte de radiação são atingidos por meio da otimização entre a exposição de todos os envolvidos pelo procedimento. Neste 
caso, tem-se como resultado imagens com qualidade diagnóstica gerando menor dose possível $(1,2,9)$. Quando aplicações médicas que fazem uso da radiação $X$ são realizadas, as blindagens são construídas considerando, além das características do material do qual a blindagem é constituída (composição e espessura), as particularidades da sala de realização dos procedimentos com raios $X$ (isto é, a geometria de irradiação), o espectro de radiação incidente, o fator de ocupação nas áreas vizinhas atrás da blindagem e os demais exames a serem realizados nesta sala $(8,10)$. Estas salas de procedimentos ou áreas onde ocorrem as exposições devem ser construídas com as blindagens corretamente dimensionadas, utilizando materiais atenuadores, que garantam que os níveis de radiação nas áreas adjacentes do local estejam de acordo com os limites de dose para o tipo de público ocupante da área em questão.

O fator característico mais importante para um material utilizado como blindagem é a curva de transmissão definida para um feixe de radiação e material específicos para auxiliar na determinação da espessura de material necessária para a atenuação $(8,11)$. Neste trabalho considera-se como base conceitual a NCRP-147 (8), recomendação internacional que define tanto curvas de transmissão para a composição média de materiais tradicionalmente usados como blindagem, quanto normas de cálculos de blindagem, tanto para barreiras primárias quanto secundárias. Os materiais que constituem uma blindagem dependem de diferentes fatores, como: tipo de radiação, energia do espectro, abertura de campo, fluência de partículas, entre outros $(8,10)$.

As dificuldades de realização de testes para a análise de novos materiais levam a busca de simulações como forma de auxílio para a otimização de blindagens. O principal objetivo deste trabalho é desenvolver um método para caracterização adicional de novos materiais para blindagem de fótons usando o Geant4 (12-14). O Geant4 é uma ferramenta computacional para simular o transporte de radiação através do método de Monte Carlo, que permite a descrição computacional dos espectros emitidos pelos equipamentos geradores de radiação $X$, dos detectores de radiação e da geometria de radiação, se assemelhando ao máximo às condições reais (15). Dentro deste contexto, este projeto tem a finalidade de utilizar a aplicação validada para gerar computacionalmente curvas e espectros.

Este trabalho se enquadra em um contexto maior, - qual visa o desenvolvimento de blindagens específicas para a proteção radiológica, numa colaboração interinstitucional. O Programa de Mestrado em Engenharia Eletrônica e Computação da Universidade Católica de Pelotas (PPGEECUCPel) é responsável pelo desenvolvimento das amostras dos novos materiais de blindagem; o Laboratório de Implantação lônica (LII) é responsável pela caracterização experimental dos materiais por meio das medidas de Rutherford Backscattering Spectrometry (RBS); e o grupo de pesquisa High
Energy Physics Simulations (HEPSim) é responsável pelo desenvolvimento da aplicação computacionalmente.

\section{Materiais e Métodos}

Este é um trabalho em andamento que já apresenta resultados consistentes. Assim sendo, serão apresentados: (i) a caracterização do material para uso em construção civil e (ii) a aplicação desenvolvida.

\subsection{O material desenvolvido}

A concepção inicial do material de blindagem foi idealizada pelo Laboratório de Resistência de Materiais da UCPel tendo como base fazer uma blindagem para fótons para uso em aplicações médicas de radiodiagnóstico. As composições das misturas são definidas como proporção normalizada dentro da mistura total de cada material. Os resultados preliminares das simulações realizadas com os materiais (doravante denominados traços) estão definidos conforme apresenta a Tabela 1.

Tabela 1. Composição da mistura e densidade dos três diferentes traços avaliados neste trabalho.

\begin{tabular}{cccc}
\multicolumn{4}{c}{ diferentes traços avaliados neste trabalho. } \\
\hline & \multicolumn{3}{c}{ Proporção na mistura } \\
\hline Material & Traço A & Traço B & Traço C \\
Cimento CPV $_{\mathrm{SiO}_{2}}$ & 0,3571 & 0,3214 & 0,3214 \\
Barita $_{\text {Basalto }}$ & 0,1071 & 0,1071 & 0,1071 \\
Água & 0,4464 & 0,4464 & 0,4465 \\
& 0,0893 & 0,0894 & 0,0893 \\
Densidade $\left(\mathrm{g} / \mathrm{cm}^{3}\right)$ & 2,51 & & \\
\hline Fonte: O autor. $(2021)$ & \multicolumn{4}{c}{2,14} & 2,44
\end{tabular}

O processo de confecção do material, bem como sua caracterização, é apresentado por Pires et al (16).

\subsection{As caracterizações experimentais}

Diferentes aspectos experimentais foram utilizados para verificar a acurácia e a confiabilidade das curvas de transmissão. Essa atividade de verificação das características dos materiais de blindagem englobou quantidades tradicionalmente utilizadas na caracterização de materiais de blindagem, como determinação da camada semirredutora (CSR) do material (18-21). Sendo a validação macroscópica da aplicação realizada através do teste da CSR e da medida de kerma (22), os quais são testes comuns utilizados em controle da qualidade em equipamentos geradores de raios $X$. Além destes, medidas de RBS (23-25) foram realizadas para análise de composição dos materiais e verificação da homogeneidade da composição para a superfície superior e inferior. Em nossa simulação, somente 0 teste de CSR e a medida de kerma foram simulados.

A CSR consiste em encontrar a espessura de material que reduza à metade a intensidade da radiação incidida pelo feixe. A medida de kerma é realizada para ser utilizada como teste de constância do equipamento gerador de raios $X$, servindo como referência para a vida útil do tubo, bem como referência de normalização do espectro simulado. 
A configuração experimental para determinação da curva de transmissão para feixe primário foi modelada de forma a simular a geometria de irradiação produzida por um equipamento de radiologia convencional para tensão de pico de $80 \mathrm{kVp}$. A Figura 1 ilustra a geometria utilizada, onde o equipamento gerador de raios $X$ é colocado a uma distância fixa do sensor de radiação. A parede é composta do material de blindagem, que é posicionado entre a fonte e o detector, próximo a superfície de entrada do detector. Quando o procedimento é realizado com filtração adicional no experimento, um filtro é colocado na saída da janela do feixe útil do equipamento gerador de radiação.

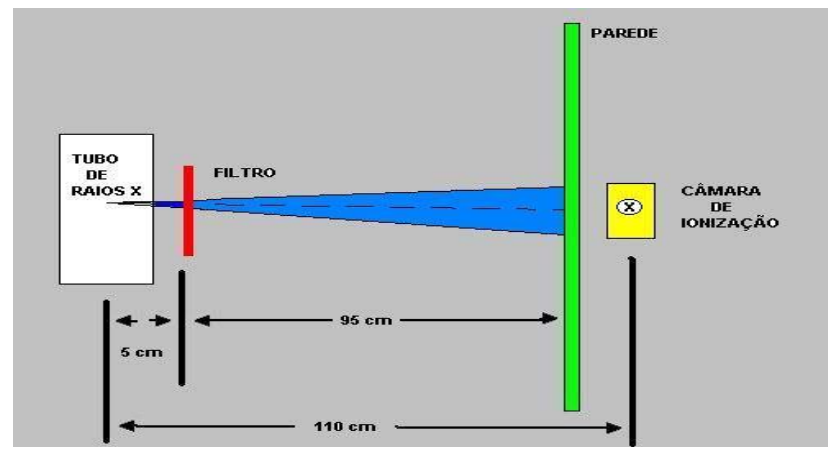

Figura 1. Esquema da geometria de irradiação para construção da curva de transmissão experimental de um material (17).

A abertura de campo utilizada para o experimento é de $10 \mathrm{~cm} \times 10 \mathrm{~cm}$ considerando as particularidades do detector utilizado. A medida de transmissão é a razão entre a medida realizada com o material de blindagem e a medida realizada sem o material de blindagem (medida de referência). Calculou-se a CSR e kerma e a mesma geometria foi repetida para diferentes espessuras de material $(0,2,4,6,8$ e $10 \mathrm{~mm}$ ), possibilitando que a transmissão seja representada em função da espessura do material para os espectros poli-energéticos emitidos pelo equipamento gerador de radiação $X$.

\subsection{Testes de homogeneidade, análise RBS e CSR}

Os testes de homogeneidade, CSR e medida de kerma foram realizados no Hospital de Clínicas de Porto Alegre, Serviço de Radiologia, Sala 4. O equipamento gerador de radiação $X$ estava em conformidade com todos os testes de Controle da Qualidade exigidos pela normativa brasileira.

$O$ teste de homogeneidade foi feito com 0 equipamento Siemens Multix Top - DR e Image Plate (IP) DRX - 1 Care Streem, usando uma placa de acrílico de $20 \mathrm{~mm}$ de espessura (PMMA). O procedimento foi realizado posicionando-se uma lâmina de $2 \mathrm{~mm}$ de espessura de PMMA na saída do colimador do tubo de raios $\mathrm{X}$ atuando como material para gerar espalhamento de maneira mais homogênea possível ao feixe primário. A imagem gerada nesta etapa será utilizada como referência comparativa para as demais imagens. Para manter esse parâmetro constante, essa lâmina espalhadora foi mantida na medida com o material de teste. Assim sendo, é possível realizar uma caracterização inicial do sistema de imagem para posterior subtração de artefatos não provindos do material de teste. Foi deixado um espaço entre a lâmina de PMMA e a saída do colimador para que a lâmina de material mais espessa possa ser imageada, com o campo útil do feixe primário fechado para cobrir toda a lâmina do material a ser avaliado (evitando as bordas). O IP foi posicionado sobre a mesa de exames com a distância foco-mesa ajustada de forma ao feixe útil cobrir todo o IP, sendo utilizada fita crepe para marcar a posição do cassete (caso houvesse algum deslocamento). A lâmina menos espessa $(2 \mathrm{~mm})$ de um dos materiais de teste foi adicionada no campo útil, realizando-se uma exposição de referência em modo semiautomático a $80 \mathrm{kVp}$, com o IP posicionado na gaveta. A técnica radiográfica de referência foi registrada ( $k V p$ e $m A s)$, sendo essa imagem não utilizada nas análises. O cassete foi posicionado sobre a mesa, na marcação prévia realizada, sendo a exposição realizada em modo manual considerando $80 \mathrm{kVp}$ de tensão de pico e 5 vezes a carga transportada no tubo (mAs) da medida de referência. Essa técnica radiográfica foi utilizada para imagear todas as lâminas do mesmo material com diferentes espessuras $(4 \mathrm{~mm}$ a $10 \mathrm{~mm})$. Cada material foi imageado seguindo o mesmo procedimento a iniciar pela medida de referência. As imagens geradas foram verificadas visualmente $e$ características como intensidade de sinal, ruído e razão sinal-ruído foram avaliadas com uso do programa Fiji.

Medidas de RBS foram realizadas no LII, localizado no Instituto de Física da Universidade Federal do Rio Grande do Sul (UFRGS). A técnica de RBS consiste na detecção dos íons retroespalhados provenientes da interação entre o feixe incidente e a amostra em estudo. Dessa forma, fornece a energia dos íons retroespalhados em função do ângulo de espalhamento. Nesta etapa do trabalho, foi utilizado um feixe incidente de $\mathrm{He}^{+}$com energia de $2 \mathrm{MeV}$. Os íons retroespalhados foram medidos por um detector de estado sólido no ângulo de retroespalhamento de $165^{\circ}$. A resolução em energia do sistema foi de aproximadamente $18 \mathrm{keV}$. A análise dos espectros obtidos foi realizada através do código de simulação PM3, disponível online (26).

O teste da CSR foi realizado com sensor externo Fluke modelo 12000WD e eletrômetro TNT 12000D, usando lâminas do material a ser testado em diferentes espessuras. $O$ sensor foi posicionado sobre a mesa no centro de campo de irradiação com distância foco-mesa ajustada em 1,0 m. O campo útil do feixe primário foi fechado de forma a cobrir toda a área útil sensível do sensor. Um suporte foi colocado na saída do colimador do tubo, permitindo o posicionamento da lâmina de material teste mais espessa. Foi selecionada a técnica radiográfica 80 $\mathrm{kVp}$ e $70 \mathrm{mAs}$, sendo esta técnica utilizada em todas as exposições para o teste de CSR. A primeira exposição de referência foi realizada sem material entre o ponto focal e a superfície de entrada do detector. A lâmina de $2 \mathrm{~mm}$ de material foi colocada no suporte e uma nova exposição foi realizada. $O$ medidor mostra, entre outras medidas, a CSR. Novas medidas foram realizadas com lâminas de diferentes 
espessuras e para todos os materiais. Estes foram base para a geração dos gráficos de CSR normalizada para comparar com a simulação.

\subsection{A aplicação desenvolvida com o Geant4}

Inicialmente, da aplicação desenvolvida foram verificados (i) os espectros teóricos para equipamentos de radiologia; (ii) a geometria de radiação e (iii) a caracterização dos materiais utilizados para blindagem.

A geometria de radiação se constitui de duas câmaras de ionização e o filtro do material da blindagem a ser simulado.

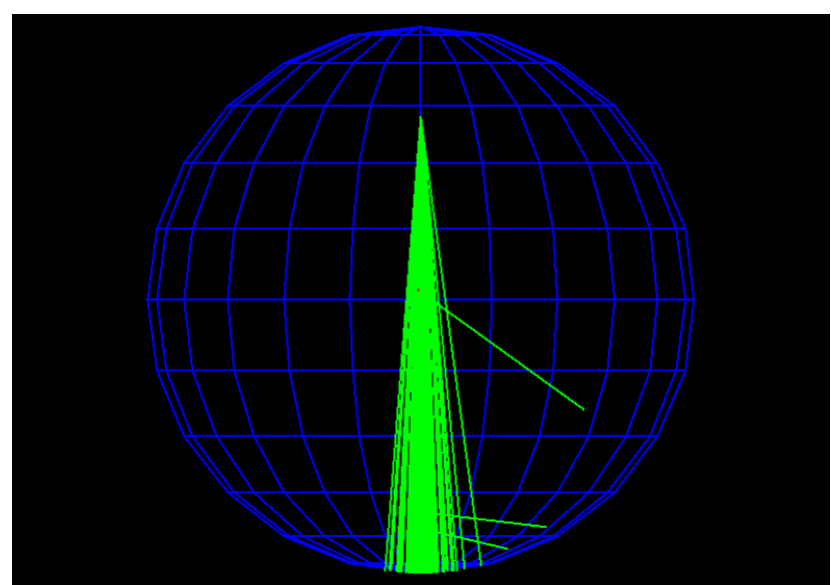

Figura 2. Figura vista da lateral, o mundo representado em azu com a fonte em cima emitindo os fótons em verde.

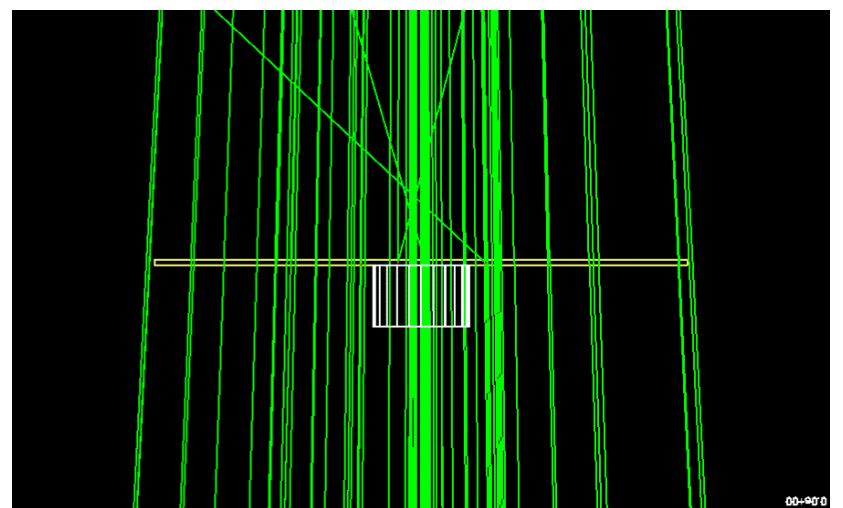

Figura 3. A câmara (em branco), e o filtro (em amarelo) vistos de lado.

A geometria foi validada por um programa de visualização do Geant4 chamado HepRapp. As simulações foram geradas considerando a lista de processos e modelos físicos Standard Option 4 recomendada pelo manual do Geant4 para simulações para fótons e elétrons de baixa energia (27). As energias de corte para geração de partículas secundárias default do Geant4 foi utilizada, entretanto, elétrons gerados no material de blindagem foram mortos imediatamente após sua criação.

Os espectros definidos para aplicação de radiologia, provindos do catálogo SRS 78 (28), foram simulados para teste de unidade da aplicação. Os espectros gerados pela aplicação foram verificados simulando $10^{5}$ histórias, com partícula geantino e nível de comunicação com o usuário em tracking 2. 0 software gnuplot foi utilizado para gerar os gráficos de frequência em função da energia da partícula emitida pela fonte.

\section{Resultados}

\subsection{Caracterização experimental dos materiais:} homogeneidade e RBS

Os resultados dos testes de homogeneidade e estimativas realizadas pelo RBS são apresentados nesta seção.

A Figura 4 apresenta os espectros de RBS obtidos na superfície (direita) e no fundo (esquerda) da amostra $A$. Os pontos pretos representam os dados experimentais e a linha azul os dados simulados. Os sinais do Bário (Ba), Cálcio (Ca), Enxofre (S), Silício (Si) e Oxigênio $(\mathrm{O})$ estão indicados na figura. Através da análise por RBS foi possível quantificar a concentração desses elementos em função da profundidade. Comparando os espectros de RBS obtidos no fundo e na superfície da amostra, observamos uma redução de aproximadamente $93 \%$ no sinal do Ba e $98 \%$ no sinal do S na superfície da amostra. Além disso, em comparação com o fundo da amostra, a superfície apresenta uma concentração de $\mathrm{Ca}$ duas vezes maior e um aumento de aproximadamente $16 \%$ na concentração de $\mathrm{O}$.

A mesma análise foi realizada nas amostras B e C e também indicaram uma sedimentação do Ba e $S$ no fundo da amostra com um aumento de $\mathrm{Ca}$ e $\mathrm{O}$ na superfície. Entretanto, o perfil de difusão do Ba da superfície para o fundo nessas amostras ocorre gradualmente.

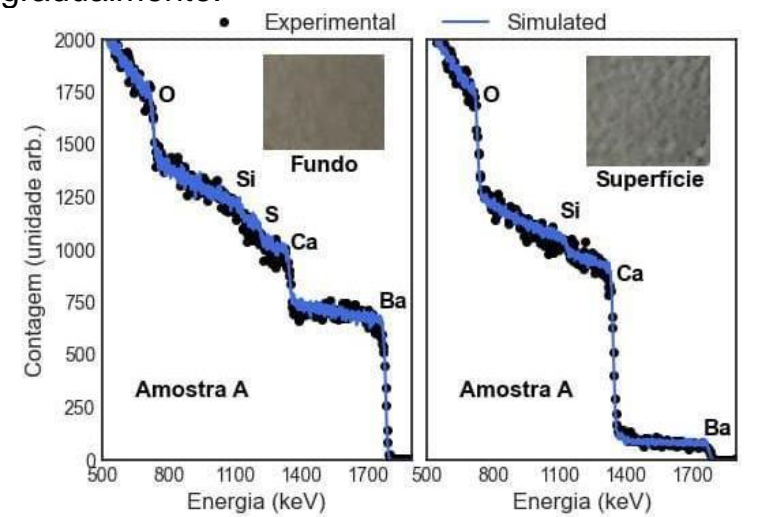

Figura 4. Espectros de RBS obtidos com um feixe incidente de 2 $\mathrm{MeV} \mathrm{He}^{+}$na superfície (direita) e no fundo (esquerda) da amostra A.

Na Figura 5 são ilustrados exemplos das imagens obtidas nos testes de homogeneidade por imageamento, sendo (a) a imagem de referência, contendo somente $2 \mathrm{~cm}$ de polimetilmetacrilato (PMMA) na saída do colimador, e, adicionada a esta, uma amostra de cada traço (de espessura de $2 \mathrm{~mm}$ ): (b) Traço A, (c) Traço B e (d) Traço C. Percebe-se: (i) a diferença na homogeneidade dos materiais, sendo o Traço $C$ visualmente o mais homogêneo, porém apresentando algumas fissuras e o Traço B o menos homogêneo, com manchas causadas por concentração de basalto e bolhas de ar; e (ii) a diferença nos tons de cinza de cada material, visto que o mesmo janelamento de cores está sendo utilizado, sendo o Traço $B$ aparentemente o mais atenuador e o Traço A mais atenuante que o Traço $C$. 

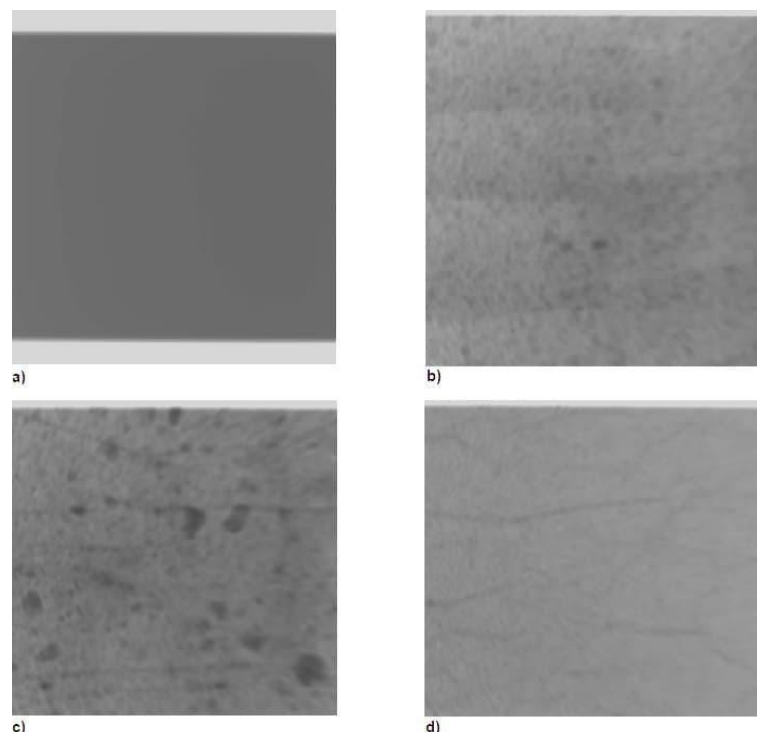

Figura 5. A imagem a) é a de referência, sem lâmina; as imagens b), c) e d) são as lâminas dos materiais Traço A, Traço B e Traço $\mathrm{C}$, respectivamente.

Tanto os resultados apresentados por RBS como os apresentados no teste de imageamento mostraram não homogeneidades significativas. As medidas de RBS permitiram a verificação de não homogeneidades em função da profundidade do material e da granularidade da mistura (especialmente as misturas que utilizam basalto). Já o teste de imageamento permite quantificar a granularidade, considerando o fator de ampliação 11,44 . Nesse caso, as imagens permitiram quantificar granularidades com dimensões entre $0,3 \mathrm{~mm}$ e 1,2 $\mathrm{mm}$ para as amostras A e B. Estes testes motivaram a equipe de desenvolvimento do material a iniciar uma nova produção, com mesma composição mas com novos procedimentos para redução da granularidade do basalto nos Traços A e B para melhor confiabilidade nos resultados de caracterização por RBS e utilizar menor quantidade de água possível na mistura, a fim de evitar a sedimentação dos elementos pesados e a precipitação dos elementos leves. Esta etapa do trabalho está em andamento.

\subsection{Testes de unidade da aplicação}

Para verificar os espectros primários gerados pela aplicação do Geant4 foi preciso usar o teste do quiquadrado de Pearson, utilizada em análise estatística de gráficos de frequência:

$$
\chi_{P}^{2}=\sum_{i=1}^{N} \frac{\left(O_{i}-E_{i}\right)^{2}}{E_{i}}
$$

onde $O_{i}$ é o valor observado de cada canal, ou seja, o valor de fluência do espectro simulado e $E_{i}$ é o valor esperado de cada canal, portanto, o valor de fluência do espectro teórico.

Para interpretar estes resultados é preciso observar o valor-p de cada espectro. Caso o valor-p esteja acima do nível de significância (NS =0,01), a hipótese nula de igualdade entre as 2 distribuições de frequências será aceita. Todos os espectros avaliados apresentaram valor-p acima do nível de significância, mostrando diferença não significativa entre o espectro teórico e o simulado.

Tabela 2. Espectros gerados pela simulação (como partículas emitidas pela fonte) comparados com os espectros catalogados com seus respectivos valores de qui-quadrado, graus de liberdade e valor-p.

\begin{tabular}{cccc}
\hline Espectro & $\chi_{P}^{2}$ & Graus de & Valor-p \\
$80 \mathrm{kVp}$ & $3,66.10^{-3}$ & 136 & 1 \\
$100 \mathrm{kVp}$ & $8,62.10^{-2}$ & 176 & 1 \\
$120 \mathrm{kVp}$ & $1,74.10^{-2}$ & 109 & 1 \\
$140 \mathrm{kVp}$ & $2,20.10^{-2}$ & 128 & 1 \\
\hline
\end{tabular}

Fonte: O autor (2021)

O operador lógico de verificação de sobreposição dos volumes foi utilizado para conferir a geometria, bem como um visualizador.

Os contadores desenvolvidos nessa aplicação foram verificados comparando os resultados gerados por eles e o resultado disponibilizado pelo tracking e control verbose do Geant4.

\subsection{Comparando CSR normalizada experimental e simulada}

Os resultados considerando o material Traço $A$ estão sendo apresentados neste trabalho para exemplificar o procedimento de caracterização por Monte Carlo. Para interpretar os dados de espessuras do traço experimentais e simuladas foi usado o teste de qui-quadrado de Pearson com a correção de Yates, sempre utilizada em frequências esperadas menores que 10 (Equação 2):

$$
\chi_{P}^{2}=\sum_{i=1}^{N} \frac{\left(\left|O_{i}-E_{i}\right|-0,5\right)^{2}}{E_{i}}
$$

O valor-p dessa amostra foi 0,49 , portanto, está acima do nível de significância definido para esta análise (NS $=0,01)$, sendo a hipótese nula de não diferença entre as curvas aceitas. Apesar dos valores médios apresentados para a transmissão normalizada serem visualmente diferentes, devido à dimensão dos desvios padrões dos dados experimentais e da flutuação estatística das simulações, essas diferenças não são significativas.

Com a ferramenta Gnumeric, a função de ajuste LOGFIT foi evocada para descrever a melhor equação, sendo aplicado o método dos "mínimos quadrados" $\left(\mathrm{R}^{2}\right)$ para definir 0 melhor ajuste logarítmico dos parâmetros $a, b$ e c, que são parâmetros de amplitude para o ajuste, esp traço que representa a espessura do traço avaliado e $S$ que pode assumir ou o valor +1 ou -1 para o conjunto de dados.

$$
T_{N}=a+b * \ln \left(S *\left(e s p_{\text {traço }}-c\right)\right)
$$

O gráfico (Figura 6) resultante do ajuste da curva de transmissão normalizada $\left(T_{N}\right)$ é definido por uma equação logarítmica que se move horizontalmente dependendo do valor de $c$ é espelhada no eixo y se $S$ $=-1$. Na Figura 6 pode-se observar os dados experimentais (como marcadores $X$ azuis) e os dados simulados (como marcadores $O$ rosa). As curvas de ajuste e os respectivos valores de $R^{2}$ também são apresentados na Figura 6 seguindo o respectivo 
código de cores: azul para dados experimentais e rosa para dados simulados. Observa-se que todos os valores de $\mathrm{R}^{2}$ foram superiores a 0,97 , o que mostra o bom ajuste da curva ao conjunto de dados utilizados.

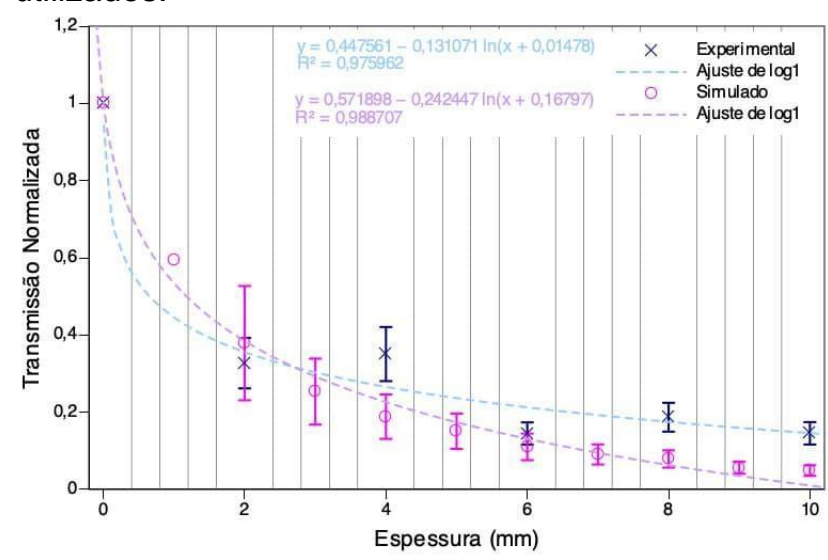

Figura 6. Transmissão normalizada em função da espessura do Traço A, com as curvas de ajuste experimental e simulada, para espectro poli-energético de $80 \mathrm{kVp}$ com alvo de tungstênio.

Já a Tabela 3 mostra os valores de camada semirredutora experimental e simulada determinados por dois métodos diferentes: utilização da curva de ajuste apresentada na Figura 6 e a utilização do método de interpolação di-log entre os dados conhecidos mais próximos ao valor da transmissão normalizada $(0,5)$ para a CSR.

Tabela 3. Valores de camada semirredutora, em mm Traço A

\begin{tabular}{lll}
\multicolumn{3}{c}{ calculados por cada método. } \\
\hline & CSR (mm Traço A) & \\
& Experimental & Simulada \\
Curva de ajuste & 0,98 & 1,18 \\
Interpolação dilog & 1,99 & 1,67 \\
\hline Fonte: O autor (2021) & &
\end{tabular}

Cabe ressaltar para as medidas experimentais que, mesmo utilizando o protocolo recomendado (para garantir os requisitos de medida em conformidade com o adequado uso do equipamento) as medidas experimentais apresentaram um alto grau de flutuação estatística para os pontos de dose medidos, evidenciado pela alteração da posição dos pontos de medida em torno do comportamento esperado. $\mathrm{O}$ equipamento utilizado apresentou dados de controle da qualidade que evidenciam uma variação estatística máxima de $4 \%$ nos testes de exatidão e reprodutibilidade de tensão de pico e de corrente de tubo. Assim sendo, essa variabilidade observada pode ser devido a não homogeneidades das lâminas do material utilizado (Traço A) e ao alto grau de atenuação do mesmo, associado a dificuldade de gerar lâminas mais finas, capazes de reproduzir múltiplos pontos de medida entre a espessura 0 a um ponto com valor de $T_{N}$ inferior a 0,5. Exatamente para possibilitar a geração destes pontos intermediários para melhor caracterização do material para uso como blindagens em radiologia diagnóstica, utilizados na simulação computacional.

A Tabela 4 mostra os valores médios de camada semirredutora de alumínio $(\mathrm{Al})$ para cada traço.

Os dados simulados apresentaram um comportamento mais conforme o esperado para curvas de transmissão de materiais homogêneos, o que fez com que os resultados de $R^{2}$ fossem ligeiramente maiores que os experimentais. Entretanto, as medidas experimentais de RBS e de homogeneidade com base na imagem das lâminas mostrou que os materiais desenvolvidos ainda não apresentam uma homogeneidade adequada (sendo o mais homogêneo o Traço C). A característica de não homogeneidade em profundidade no material resultou em um comportamento mais discrepante com relação aos dados simulados. Essa afirmação pode ser verificada com as medidas diretas de CSR equipamento em $\mathrm{mm}$ Al geradas pelo medidor para os Traços (A, B e C) desenvolvidos neste trabalho, apresentados na Tabela 4. Observa-se uma tendência de aumento do valor equivalente da espessura de alumínio com o aumento da espessura do traço avaliado, entretanto são observados valores não esperados (grafados em negrito) como os apresentados na Tabela 4. Estes valores podem ser frutos das não homogeneidades verificadas nas amostras, das rugosidades da superfície da amostra e/ou da flutuação do espectro emitido (mesmo que essa já esteja dentro dos padrões normativos nacionais).

Tabela 4. CSR equivalente, em $\mathrm{mm} \mathrm{Al}$, para cada traço avaliado, considerando todas as espessuras.

\begin{tabular}{cccc}
\multicolumn{4}{c}{ avaliado, considerando todas as espessuras. } \\
\hline Espessura $(\mathrm{mm})$ & Traço A & Traço B & Traço C \\
0 & 3,10 & 3,10 & 3,10 \\
2 & 4,57 & 4,93 & 4,57 \\
4 & $\mathbf{4 , 2 7}$ & $\mathbf{6 , 9 3}$ & $\mathbf{4 , 1 0}$ \\
6 & $\mathbf{5 , 9 7}$ & 5,67 & 4,80 \\
8 & $\mathbf{5 , 6 3}$ & $\mathbf{5 , 4 3}$ & $\mathbf{6 , 8 7}$ \\
10 & 6,10 & $\mathbf{5 , 2 3}$ & $\mathbf{5 , 8 0}$ \\
\hline
\end{tabular}

Fonte: O autor (2021)

Os passos de caracterização experimental dos Traços, bem como a simulação computacional, foram importantes na definição de critérios de qualidade para os materiais desenvolvidos, sendo possível verificar a existência de não homogeneidades nos Traços e a influência destas nas medidas de transmissão.

\section{Conclusões}

A eficácia do material desenvolvido para blindar a radiação $X$ foi aferida por medidas de transmissão do material, para diferentes espessuras (de $2 \mathrm{~mm}$ a $10 \mathrm{~mm}$ ) e energias de feixe incidente $(60 \mathrm{kVp}, 90 \mathrm{kVp}$ e $125 \mathrm{kVp}$ ). Os resultados indicam que para a maior energia avaliada (125 kVp) a espessura de 16,70 mm do traço A é suficiente para barrar $95 \%$ da radiação $\mathrm{X}$, conforme recomendado pela Portaria Federal $\mathrm{n}^{\circ}$ 453 da Anvisa (29) e atendendo ao critério restritivo para uso em áreas intervencionistas, ao artigo 51 item II da Resolução da Diretoria Colegiada $n^{\circ} 330$. Foi utilizada ainda a revogada Portaria Federal $n^{\circ} 453$, pois esta forneceu um parâmetro mais específico referentes ao material. Posteriormente, estas características de transmissão foram colocadas a prova em cálculos realistas de blindagem aplicados à radiologia, seguindo critérios da $\operatorname{RDC} n^{\circ} 330$ (1).

Este é um trabalho em andamento, mas que já possui resultados preliminares interessantes de validação da CSR mostrando a consistência dos dados. Todavia os resultados também trouxeram 
críticas ao material desenvolvido. Com base nessas críticas estamos planejando executar as seguintes atividades futuras: (i) melhorar a homogeneidade do material elaborado com padronização ainda maior do processo de produção, (ii) realizar todos os testes de densidade e resistência do material para garantir que sejam adequados para construção civil, (iii) realizar novos testes de homogeneidade e RBS, e (iv) fazer medidas de transmissão para radiologia $e$ radioterapia.

\section{Agradecimentos}

Os autores agradecem ao Conselho Nacional de Desenvolvimento Científico e Tecnológico (CNPq) pelo suporte parcial com a Bolsa de Pós-Doutorado Sênior (168442 / 2018-3).

\section{Referências}

1. Agência Nacional de Vigilância Sanitária (Brasil) [homepage on the Internet]. Resolução da Diretoria Colegiada - RDC no 330. Diário Oficial da União, 20/12/2019. Disponível em: <https://www.in.gov.br/web/dou/-/resolucao-rdc-n-330-de-20de-dezembro-de-2019-235414748?inheritRedirect=true>. Acesso em: 09/03/2021

2. Comissão Nacional de Energia Nuclear (CNEN-NN-3.01). Diretrizes Básicas de Proteção Radiológica. 2005.

3. Archer, BR. History of the shielding of diagnostic $x$-ray facilities. Health physics 69.5. 1995. p. 750-758.

4. Edwards M, Bushong SC, Dalrymple GV, Kereiakes, JG, Gibbs, SJ. Implementation of the Principle of As Low As Reasonably Achievable (ALARA) for Medical and Dental Personnel. Technical report no ${ }^{\circ}$ 107, National Council on Radiation Protection and Measurements, 1990. Disponível em: $<$ https://ncrponline.org/publications/reports/ncrp-reports107/>. Acesso em: 10/03/2021.

5. Hansson, SO. ALARA: What is reasonably achievable? Radioactivity in the Environment. Vol. 19. Elsevier, 2013. p. 143-155.

6. Quinn AD, Taylor CG, Sabharwal T, Sikdar T. Radiation protection awareness in non-radiologists. The British journal of radiology, 70(829), 102-106, 1997

7. Hendee WR, Edwards FM. ALARA and an integrated approach to radiation protection. In Seminars in nuclear medicine (Vol. 16, No. 2, pp. 142-150). WB Saunders, 1986.

8. Archer BR, Gray JE, Dixon RL, Eide WR, Hubbard LB, Kearsley EE, et al. Structural Shielding Design for Medical X-Ray Imaging Facilities. Technical report no 147, National Council on Radiation Protection and Measurements, 2004

9. Xavier AM, Moro JT, Heilbron PF. Princípios Básicos de Segurança e Proteção Radiológica, revisada. Universidade Federal do Rio Grande do Sul, 2006.

10. Santos RR, Real JV, Friedrich $B Q$, da Luz RM, da Silva AM SHIELD 1.0: desenvolvimento de um programa de cálculo de blindagem em radiodiagnóstico. Revista Brasileira De Física Médica, 7(2). 2015. p. 75-78.

11. Archer BR, Thornby JI, Bushong SC. Diagnostic x-ray shielding design based on an empirical model of photon attenuation. Health physics, v. 44, n. 5, p. 507-517, 1983.

12. S. Agostinelli, J. Allison, K. Amako, J. Apostolakis, et al. Geant4 - a simulation toolkit. Nuclear instruments and methods in physics research section A: Accelerators, Spectrometers, Detectors and Associated Equipment, 506(3):250-303, 2003.

13. J. Allison, K. Amako, J. Apostolakis, et al. Geant4 developments and applications. IEEE Transactions on nuclear science, 53(1):270-278, 2006.

14. J. Allison, K. Amako, J. Apostolakis, et al. Recent developments in Geant4. Nuclear Instruments and Methods in Physics Research Section A: Accelerators, Spectrometers, Detectors and Associated Equipment, 835:186-225, 2016.

15. Geant4 Collaboration - Geant4 Homepage - Book For Application Developers. Disponível em: <https://geant4userdoc.web.cern.ch/UsersGuides/ForApplicationDeveloper/ht $\mathrm{ml} />$. Acesso em: 10/03/2021.

16. Pires MM, do Nascimento CD, Souza EG, Kruger K, Hoff G. Utilização de sulfato de bário como constituinte de concreto para blindagem de salas de radiologia, Revista Matéria. 2021, no prelo.

17. Firmino, SF. Transmissão e fluência de fótons na área de radiodiagnóstico para diferentes configurações de feixes e blindagens [dissertação]. Porto Alegre (RS): Universidade Federal do Rio Grande do Sul; 2010.

18. Johns HE, Cunningham JR. The physics of radiology. Illinois, USA; 1983.

19. Erik AA, Kavaz E, Ilkbahar S, Kara U, Erik CE, Tekin HO. Structural and photon attenuation properties of different types of fiber post materials for dental radiology applications. Results in Physics, v. 13, 2019.

20. Waly ESA, Al-Qous GS, Bourham MA. Shielding properties of glasses with different heavy elements additives for radiation shielding in the energy range $15-300 \mathrm{keV}$. Radiation Physics and Chemistry, v. 150, p. 120-124, 2018.

21. Seenappa L, Manjunatha HC, Chandrika BM, Chikka H. A study of shielding properties of $x$-ray and gamma in barium compounds. Journal of Radiation Protection and Research [internet]. 2017 Mar 30;42(1):26-32. Available from: http://dx.doi.org/10.14407/jrpr.2017.42.1.26.

22. Thomas, DJ. ICRU report no 85: fundamental quantities and units for ionizing radiation, Radiation Protection Dosimetry, Volume 150, Issue 4, July 2012.

23. Meng IM, Azman NZ. Polymer Composites and Nanocomposites for x-rays shielding. Springer, 2020.

24. Olukotun SF, Gbenu ST, Oladejo OF, Sayyed MI, Tajudin SM, Amosun $A A$, et al. Investigation of gamma ray shielding capability of fabricated clay-polyethylene composites using EGS5, XCOM and Phy-X/PSD. Radiation Physics and Chemistry, v. 177, p. 109079, 2020.

25. Rodriguez, DG, Bastos RO, Ikeoka RA, Appoloni CR, Bandeira AM. Gamma-ray spectrometry in the characterization of diverse-geometry archaeological ceramics. Archaeometry, 2020.

26. G.G. Marmitt, PowerMEIS simulation code, 2019. Disponível em: <http://tars.if.ufrgs.br/>. Acesso em: 10/03/2021.

27. Geant4 Collaboration - Geant4 Homepage - PhysicsListGuide - Docs - Electromagnetic physics constructors - EM Opt4. Disponível em: <https://geant4userdoc.web.cern.ch/UsersGuides/PhysicsListGuide/html/elec tromagnetic/Opt4.html\#em-opt4>. Acesso em: 24/02/2021.

28. Cranley, K. Catalogue of diagnostic x-ray spectra and other data. The Institute of Physics and Engineering in Medicine Report, 1997.

29. Agência Nacional de Vigilância Sanitária (Brasil). Diretrizes de proteção radiológica em radiodiagnóstico médico e odontológico. Portaria no 453. Diário oficial da União, 01/06/1998.

\section{Contato:}

Natalia Amaral Lourenço

Instituto de Física da UFRGS

Av. Bento Gonçalves, 9500 - Agronomia - Instituto de

Física - Porto Alegre, RS

nt.aa@hotmail.com 